\title{
Determination of the order of Kinetics of a DTA Curve by using the Concept of Skewness
}

\author{
BIKASH KUMAR SARKAR ${ }^{1 *}$, INDRANIL BHATTACHARYYA ${ }^{2}$, \\ ANANDA SARKAR ${ }^{2}$ and PARTHA SARATHI MAJUMDAR ${ }^{2}$

\begin{abstract}
'Department of Physics, Mrinalini Datta Mahavidyapith, Birati, Kolkata-700051, West Bengal, India. 2Department of Physics, Acharya Prafulla Chandra College, New Barrackpore, Kolkata-700131, West Bengal, India.

*Corresponding author E-mail: biku.mdm @gmail.com
\end{abstract} \\ http://dx.doi.org/10.13005/ojc/360632
}

(Received: November 11, 2020; Accepted: December 12, 2020)

\section{ABSTRACT}

In the present paper, a novel way of finding out the order of kinetics of differential thermal analysis (DTA) curves by using the concept of skewness $\left(\mathrm{S}_{k}\right)$ has been investigated. It is found that for a particular DTA peak, skewness is a function of both the order of kinetics and the quantity, $u_{m}=\frac{E}{R T_{m}}$ First order DTA peaks are characterized by negative skewness whereas those for the second order are characterized by positive skewness. Therefore, skewness can be used as an indicator of the order of kinetics of a DTA peak. We have evaluated and compared the orders of kinetics of some reported DTA peaks using the concept of skewness and found that the resulting values of the orders of kinetics are in fair agreement with those reported in literature.

Kewords: Activation energy, Skewness, Orders of kinetics, Differential thermal analysis.

\section{INTRODUCTION}

Differential thermal analysis (DTA) is a method which is dependent on the observation of chemical and physical changes in a given sample occurred due to the change of temperature in a predetermined manner ${ }^{1,2,3}$. The curves obtained by such analysis are called DTA curves. Three important kinetic parameters, namely order of kinetics $(n)$, activation energy $(E)$ and pre-exponential factor $(A)$ can be evaluated from these DTA curves for different energetic materials. Since, the shape of the peak of the DTA curves and the values of kinetic parameters are indicators of the composition of the sample, these curves are widely utilized for the qualitative identification of inorganic and organic substances.

Order of kinetics $(n)$ is one of the important kinetic parameters of a DTA curve. It is related to the symmetry of a DTA curve. From the statistical point of view, the symmetry of a DTA curve should be determined by using a longer portion of the curve. This symmetry can be characterized by the skewness $\left(\mathrm{S}_{k}\right)$ of DTA peaks. In the present study,

This is an Open Access article licensed under a Creative Commons license: Attribution 4.0 International (CC- BY). Published by Oriental Scientific Publishing Company @ 2018

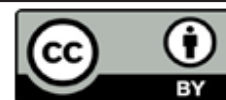


skewness of DTA curves resulted from kinetic order model (KOM) has been investigated. A novel attempt has been made to relate the skewness of a DTA peak with its order of kinetics. Finally, the present technique is applied to determine the orders of kinetics $(n)$ of some reported DTA curves. To best of our knowledge, DTA curve has not been analysed by using the concept of skewness.

\section{Methodology}

\section{Concept of skewness}

Skewness ${ }^{4,5}$ is the degree of asymmetry of a distribution. If the frequency curve of a distribution has a longer tail to the right of the maximum than to the left of the distribution, it is said to be skewed to the right or has positive skewness. If the reverse happens, it is said to be skewed to left or negatively skewed. A symmetric distribution has zero skewness. Different types of skewness are shown in Figure 1.

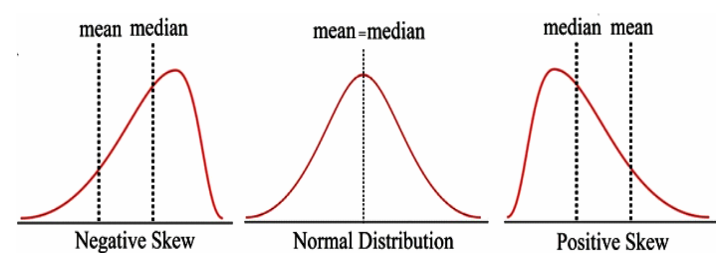

Fig. 1. Different types of skewness

The $r^{\text {th }}$ order moment ${ }^{4,5}$ about an arbitrary origin is defined as

$m_{r}=\frac{1}{N} \sum_{i=1}^{n}\left(x_{i}-\bar{x}\right)^{r}$

where, $x_{i}$ and $\bar{x}$ are respectively $i^{\text {th }}$ observed value and mean value of the distribution and $N$ is the number of data points.

Skewness, actually, indicates the lack of symmetry. The odd order central moments are positive for positively skewed distribution, zero for symmetric distribution and negative for negatively skewed distribution.

The $3^{\text {rd }}$ order central moment about the mean is give by

$$
m_{3}=\frac{1}{N} \sum_{i=1}^{n}\left(x_{i}-\bar{x}\right)^{3}
$$

It can be shown that for positively skewed distribution, $m_{3}$ is positive whereas for negatively skewed distribution $m_{3}$ is negative. For this reason, $m_{3}$ is taken as the moment measure of skewness. $m_{3}$ increases with the increase of asymmetry. As $m_{3}$ depends on the unit of measurement, it is not a pure number.

The dimensionless form of skewness is given by ${ }^{4,5}$

$s_{k}=\frac{m_{3}}{\delta^{3}}$

where, $\delta$ is the standard deviation.

\section{Theoretical analysis of DTA curves recorded under a linear heating scheme}

The rate of change of concentration for a solid state reaction can be expressed $a s^{6}$

$$
-\frac{d x}{d t}=A \exp \left(-\frac{E}{R T}\right) \frac{x^{n}}{x_{0}^{n-1}}
$$

where, $x_{0}$ is the initial concentration, $x$ is the concentration at time $t, A$ is the pre-exponential factor and $R$ is the universal gas constant.

The temperature deviation $\Delta T$ for a DTA curve from horizontal base line is given by

$$
\Delta T=-\beta \frac{d x}{d t}
$$

where, $\beta$ is a proportionality constant.

For a linear heating scheme, $\mathrm{T}=\mathrm{T}_{0}+\phi t$

where, $T_{0}$ and $T$ are respectively initial temperature and temperature at time $t$ and $\phi$ is the constant heating rate.

From equations (4), (5) and (6), it follows that

$$
\begin{aligned}
& \Delta T=-A \beta \exp \left(-\frac{E}{R T}\right)\left[1+(n-1) \frac{A}{\phi} \int_{T_{0}}^{T} \exp \left(-\frac{E}{R T}\right) d T\right]^{-\frac{n}{n-1}}(\mathrm{n} \neq 1) \\
& \Delta T=-A \beta \exp \left(-\frac{E}{R T}\right) \exp \left[\frac{-A}{\phi} \int_{T_{0}}^{T} \exp \left(-\frac{E}{R T}\right) d T\right] \begin{array}{r}
(\mathrm{n}=1) \\
(8)
\end{array}
\end{aligned}
$$

At peak temperature, $T=T_{m}$ the deviation $\Delta T$ of the DTA curve from the base line is extremum and

$$
\left[\frac{d}{d T}(\Delta T)_{T=T_{m}}\right]=0
$$

From equations (6)-(8), it is possible to write 
$1+(n-1) \frac{A}{\phi_{T_{0}}^{T}} \int_{T_{0}} \exp \left(-\frac{E}{R T}\right) d T=\frac{n A R T_{m}^{2}}{\phi E} \exp \left(-\frac{E}{R T_{m}}\right) \begin{array}{r}(\mathrm{n} \neq 1) \\ (10)\end{array}$

and

$\frac{\phi E}{R T_{m}^{2}}=A \exp \left(-\frac{E}{R T_{m}}\right)$

The integral $\int_{T_{0}}^{T} \exp \left(-\frac{E}{R T}\right) d T$ is commonly known as temperature integral ${ }^{1}$. The lower limit $\mathrm{T}_{0}$ of the above integral can be replaced by zero for which

$\int_{T_{0}}^{T} \exp \left(-\frac{E}{R T}\right) d T \approx \int_{0}^{T} \exp \left(-\frac{E}{R T}\right) d T$

It can be shown that

$\begin{aligned} \int_{0}^{T} \exp \left(-\frac{E}{R T}\right) d T & =\frac{E}{R} \frac{E_{2}(u)}{u} \\ \text { with } u_{m} & =\frac{E}{R T_{m}}, E_{2}(\mathrm{u}) \text { is the second }\end{aligned}$ exponential integral ${ }^{7}$.

Using equations (7), (8), (10) and (11), it follows that

$\frac{\Delta T}{(\Delta T)_{m}}=\exp \left(u_{m}-u\right)\left[1-\frac{n-1}{n} F\left(u, u_{m}\right)\right]^{-\frac{n}{n-1}}(\mathrm{n} \neq 1)$

$\frac{\Delta T}{(\Delta T)_{m}}=\exp \left[u_{m}-u+F\left(u, u_{m}\right)\right]$

where, $u_{m}=\frac{E}{R T_{m}}$.

$\mathrm{F}\left(\mathrm{u}, \mathrm{u}_{\mathrm{m}}\right)$, can be written as

$F\left(u, u_{m}\right)=u_{m}^{2} \exp \left(u_{m}\right)\left\lceil\frac{E_{2}\left(u_{m}\right)}{u_{m}}-\frac{E_{2}(u)}{u}\right\rceil$

$\Delta T_{m}$ is the maximum value of $\Delta T$.

\section{Calculation of skewness for a DTA curve}

The curve obtained by plotting $T$ as independent variable and $\Delta T$ as dependent variable represents a DTA curve. Therefore, from equations (7) and (8), the skewness $S_{k}(x)$ Corresponding to $x=\frac{\Delta T}{(\Delta T)_{m}}$ for a DTA curve with a particular order of kinetics $\mathrm{n}$ is given by

$S_{k}(x)=\frac{c}{d}$

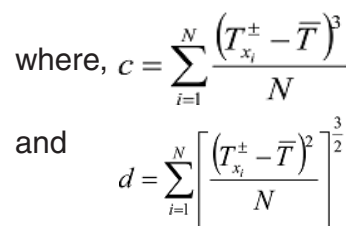

Here, $\mathrm{T}^{ \pm \mathrm{i}}$ is the temperature in DTA curve corresponding to $x=\frac{\Delta T}{(\Delta T)_{m}} \bar{T}$ is the average of all temperatures taken into consideration. Equation (18) can be derived by the concept of equation (2). For a particular value of $n$, one can calculate $\mathrm{T}^{-}$and $\mathrm{T}^{+}$for an arbitrary value of $x$ from equations (14) and (15) (for $n \neq 1$ and $n=1$ ) by using an iterative technique ${ }^{8}$. $\mathrm{T}^{-}$and $\mathrm{T}_{x}^{+}$stand respectively for temperatures in the left and right halves of the peak. $S_{k}(x)$ can be evaluated by using equation (17) for different values of $X$.

\section{RESULTS AND DISCUSSION}

From the discussions in the previous section, it is evident that $S_{k}(x)$ can be evaluated by using equation (17) for different values of $x$. To check the validity of the concept adopted, $S_{k}(0.5)$ is calculated for some reported9, 10 DTA curves. By evaluating the temperatures $T_{0.50}^{-}, T^{-}{ }_{0}$. ${ }_{55}, T_{0.60}^{-}, T_{0.65}^{-} T_{0.70}, T_{0.75}, T_{0.80}^{-}, T_{0.85}, T^{-}{ }_{0.90}, T_{0.95}^{-}$, $T_{0.95}^{+} T_{0.90}, T_{0.85}^{+}, T_{0.80}^{+}, T_{0.75}^{+}, T_{0.70}^{+}, T_{0.65}^{+}, T_{0.60}^{+}, T_{0.55}^{+}$ $\mathrm{T}^{+}{ }_{0.50}$ and putting the values of these temperatures in equations (18-19), $S_{k}(0.5)$ has 5 been calculated with the help of equation (17). From the calculation, it is observed that for a particular value of $x, S_{k}(0.5)$ depends both on $n$ and $u_{m}=\frac{E}{R T_{m}}$ and varies from -0.08 to -0.142 as $u_{m}$ changed from 10 to 100 for first order kinetics $(n=1)$. Again for $n=2$, the value of $S_{k}(0.5)$ varies from 0.0075 to 0.009 for $10 \leq u_{m} \leq 100$ $\leq u_{m} \leq$. It is, however, observed that the dependence of $S_{k}(0.5)$ on $u_{m}$ is weaker than the dependence of $S_{k}(0.5)$ on $\mathrm{n}$. In Fig. 2, the variation of $S_{k}(0.5)$ with $\mathrm{mu}$ is shown for $1=\mathrm{n}, 1.5$ and 2 . It is evident from Fig. 2 that $S_{k}(0.5)$ decreases with increase in $u_{m}$ for all values of $\mathrm{n}$. Therefore, $S_{k}(0.5)$ depends both on $n$ and $u_{m}$. Skewness has also been evaluated for two other values of $x$ namely $6667.0=x$ and $8.0=x$ to study the consistency of the results. These are denoted by $S_{k}(0.6667)$ and $S k(0.8)$. 


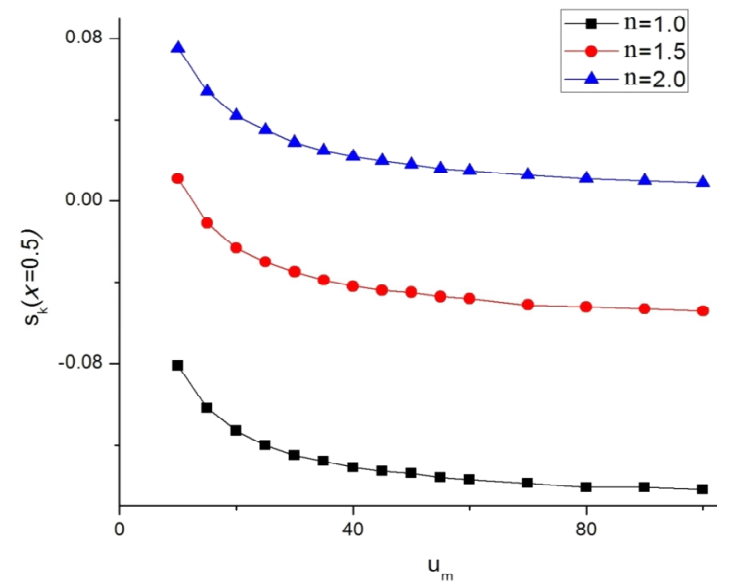

Fig. 2. Variation of skewness $(x=0.5)$ with $u_{m}$

For a particular value of $x$ and $n$, the value of $S_{k}(x)$ is not unique. Therefore, for a particular value of $x$ and $n$, the mean value of $S_{k}(x)$ for $10 \leq u_{m} \leq 100$ is calculated. The mean value of $S_{k}(x)$ for a particular value of $\mathrm{n}$ for the range $10 \leq u_{m} \leq 100$ is given by

$\bar{S}_{k}(x)=\frac{1}{90} \int_{10}^{100} S_{k}\left(x, u_{m}\right) d u_{m}$

To increase the accuracy of the evaluation of the integral in equation (20), the range (10, $100)$ of the integration is divided into a number of sub-ranges $(10,20),(20,30),(30,40),(40$, $50),(50,60),(60,70),(70,80),(80,90)$ and $(90$, $100)$. The integral over each sub-range has been carried out by using 16-point Gauss-Legendre quadrature $^{7}$. In Fig. 3 , the variation of $\bar{S}_{k}(0.5)$ with order of kinetics $\mathrm{n}$ for $21 \leq n \leq$ is depicted. It is noticed from Fig. 3 that the sign of $\bar{S}_{k}(0.5)$ changes around $n=1.7$ to 1.8. Therefore, as $n$ varies from first order to second order, DTA curve gets transposed from symmetric to asymmetric type. Skewness, therefore, is both a qualitative and quantitative indicator of the symmetry of a DTA curve. $\bar{S}_{k}(x)$ has also been calculated for $x=0.6667$ and $x=0.8$. The variations of $\bar{S}_{k}$ $(0.6667)$ and $\bar{S}_{k}(0.8)$ with $u_{m}$ are also shown in Fig. 3. $\bar{S}_{k}(0.6667)$ and $\bar{S}_{k}(8.0)$ also display the change of sign. The variations of $\bar{S}_{k}(0.6667)$ and $\bar{S}_{k}(0.8)$ with $\mathrm{u}_{\mathrm{m}}$ for $n=1,1.5$ and 2 are respectively presented in Fig. 4 and in Fig. 5. Finally, by using the technique of least square fitting ${ }^{4} \bar{S}_{k}(0.5), \bar{S}_{k}$ $(0.6667)$ and $\bar{S}_{k}(0.8)$ are expressed as a function of order of kinetics $\mathrm{n}$ as follows:

$\bar{S}_{k}(0.5)=-0.3783+0.3086 \mathrm{n}-0.0526 \mathrm{n}^{2}$

$\bar{S}_{k}(0.6667)=-0.2440+0.2002 \mathrm{n}-0.0345 \mathrm{n}^{2}$
$\bar{S}_{k}(8.0)=-0.1228+0.1135 n-0.0244 n^{2}$

Using equations (21)-(23), the order of kinetics $n$ of DTA peaks has been calculated.

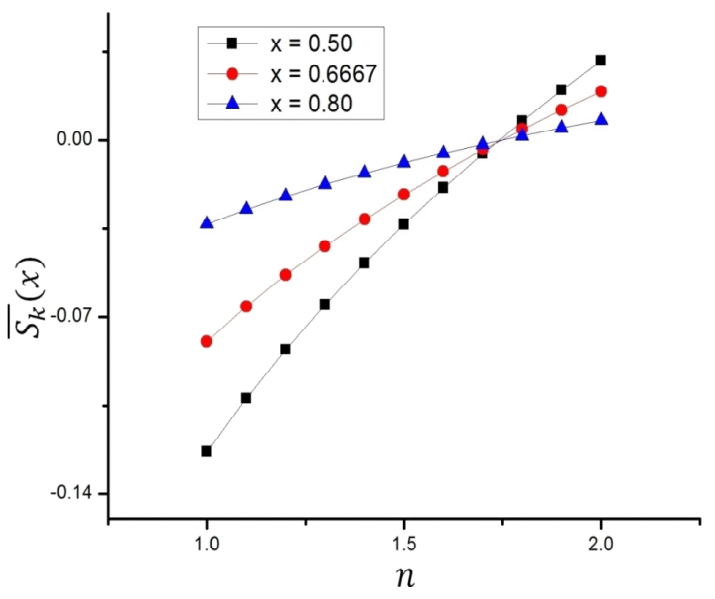

Fig. 3. Variation of average value of skewness $(x=0.5,0.6667,0.8)$ with order of kinetics $(n)$

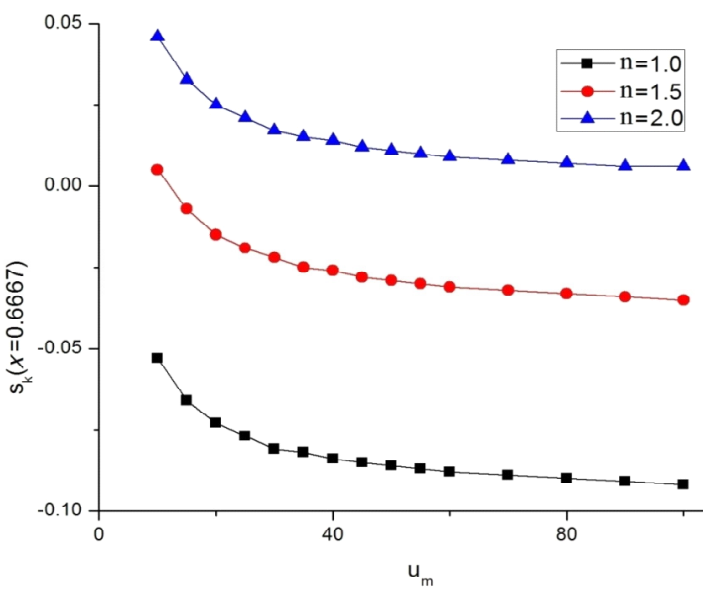

Fig. 4. Variation of skewness $(x=0.6667)$ with $u_{m}$

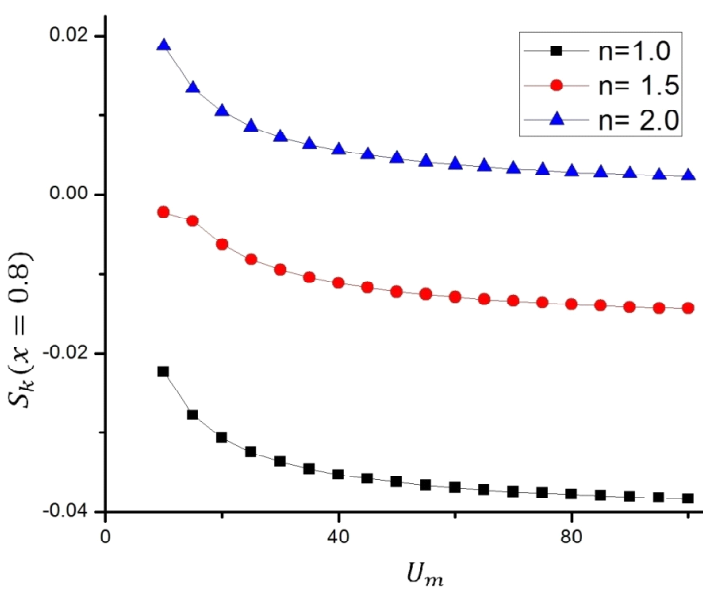

Fig. 5. Variation of skewness $(x=0.8)$ with $u_{m}$ 
Calculation of order of kinetics by using skeewness from reported DTA curves

Some reported ${ }^{9,10}$ DTA curves of Georgia kaolinite, Eureka halloysite, RDX and TNT are considered. $S_{k}(x)$ for different values of $x$ has been computed from these curves. The values of $S_{k}(0.5), S_{k}(0.6667)$ and $S_{k}(0.8)$ for these DTA peaks computed have been presented in Table 1. The orders of kinetics of these peaks as determined from their skewness (equations 21-23) have also been displayed in Table 1. It is observed that the mean value of the orders of kinetics of these peaks is in fair agreement with corresponding values reported in the literature ${ }^{9,10}$.

Table 1: Determination of the orders of kinetics of some reported DTA peaks ${ }^{9,10}$ using skewness

\begin{tabular}{|c|c|c|c|c|c|c|c|c|c|c|c|}
\hline \multirow[t]{2}{*}{ Material } & \multirow[t]{2}{*}{$\begin{array}{l}\text { Heating } \\
\text { Rate } \\
\left({ }^{\circ} \mathrm{Cmn}^{-1}\right)\end{array}$} & \multicolumn{3}{|c|}{$\begin{array}{l}\text { Kinetic parameters of } \\
\text { the peaks as reported } \\
\text { in the literature } \\
9,10\end{array}$} & \multirow[b]{2}{*}{$\begin{array}{c}S_{k} \\
(0.5)\end{array}$} & \multirow{2}{*}{$\begin{array}{c}S_{k}(x) \\
S_{k} \\
(0.6667)\end{array}$} & \multicolumn{3}{|c|}{$\begin{array}{c}n \text { calculated from } \\
\text { eqns. }(21-23)\end{array}$} & \multirow[b]{2}{*}{$\begin{array}{c}S_{k} \\
(0.8)\end{array}$} & \multirow[t]{2}{*}{ Mean $n$} \\
\hline & & $E$ & $\begin{array}{c}A \\
(k c a l)\end{array}$ & $\begin{array}{c}n \\
\left(\sec ^{-1}\right)\end{array}$ & & & $\begin{array}{c}S_{k} \\
(0.8)\end{array}$ & $\begin{array}{c}S_{k} \\
(0.5)\end{array}$ & $\begin{array}{c}S_{k} \\
(0.6667)\end{array}$ & & \\
\hline Georgia kaolinite $^{9}$ & 6 & 36.51 & $3.33 \times 10^{6}$ & 1 & -0.115 & -0.066 & -0.031 & 1.01 & 1.1 & 1.04 & $1.05 \pm 0.04$ \\
\hline Eureka halloysite $^{9}$ & 6 & 37.76 & $5.05 \times 10^{7}$ & 1 & -0.119 & -0.068 & -0.032 & 1.03 & 1.08 & 1.02 & $1.04 \pm 0.03$ \\
\hline $\mathrm{RDX}^{10}$ & 15 & 46.58 & $7.10 \times 10^{17}$ & 0.96 & -0.142 & -0.082 & -0.038 & 0.93 & 0.98 & 0.9 & $0.94 \pm 0.05$ \\
\hline $\mathrm{TNT}^{10}$ & 6 & 21.95 & $6.78 \times 10^{5}$ & 1.65 & 0.0003 & -0.0004 & -0.0003 & 1.7 & 1.74 & 1.74 & $1.73 \pm 0.02$ \\
\hline $\mathrm{TNT}^{10}$ & 10 & 20.99 & $2.87 \times 10^{5}$ & 1.75 & 0.0163 & 0.009 & 0.004 & 1.85 & 1.85 & 1.87 & $1.86 \pm 0.01$ \\
\hline
\end{tabular}

\section{CONCLUSION}

In the present paper, the skewness of DTA curves has been studied. Skewness of a first order DTA peak is negative and that of a second order DTA peak is positive. Furthermore, as the sign of skewness changes as order of kinetics of a DTA curve changes from first order to second order, it is also a qualitative indicator of the symmetry of a DTA curve. Since skewness is evaluated by using a longer portion of a DTA curve, it can be utilized as a reliable indicator of the order of kinetics of the curves considered. We have evaluated orders of kinetics of some reported DTA peaks and encouraging results have been obtained. This concept of finding out the order of kinetics of DTA curve can serve as a powerful method.

\section{ACKNOWLEDGMENT}

We acknowledge Dr. Saktibrata Bhowmik, Principal, Acharya Prafulla Chandra College for providing the facilities to carry out the work and fruitful discussions.

\section{Conflict of interest}

The authors have no conflict of interest to declare.

\section{REFERENCES}

1. Chen, R.; Krish, Y. Analysis of Thermally Stimulated Processes, Pergamon Press, Oxford., 1981.

2. Chen, R.; Mckeever, S. W. S. Theory of Thermoluminescences and Related Phenomena, World Scientific, Singapore., 1997.

3. Balarin, M. Journal of Thermal Analysis., 1979, 17, 319-332.

4. Das, N. G. Statistical Methods, McGraw Hill Education (India) Private Limited., 2012.

5. Spigel, M. R.; Stephens, I. J. Theory and Problems of Statistics, $3^{\text {rd }}$ Edition, Tata McGraw Hill Publication Company, New
Delhi., 2000.

6. Luo, K. M. Thermochim. Acta., 1995, 255, 241-254.

7. Abramwitz, M.; Stegun, J. A. Hand Book of Mathematical Functions, Dover, New York., 1965.

8. Balagurusamy, E. Numerical Methods, Tata McGraw Hill Publication Company, New Delhi., 2000, Chapter 6.

9. Yang, R. T.; Steinberg, M. Anal. Chem., 1977, 49, 998-1001.

10. Huang, C. C.; Wu, T.S. Thermochim. Acta., 1992, 204, 239-250. 